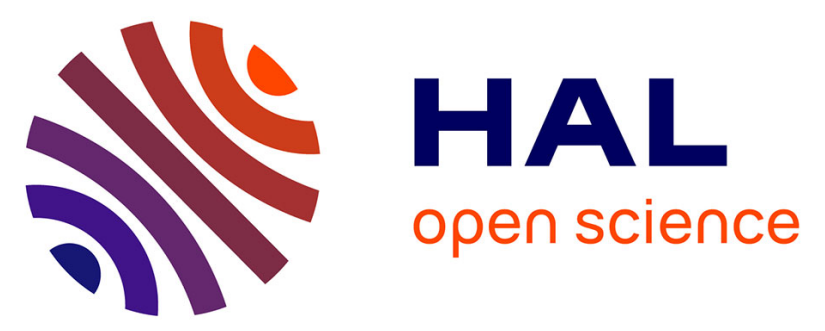

\title{
Echo-Less Photoconductive Antenna Sources for High-Resolution Terahertz Time-Domain Spectroscopy
}

Kenneth Maussang, Anthony Brewer, José Palomo, Jean-Michel Manceau, Raffaele Colombelli, Isabelle Sagnes, Juliette Mangeney, Jérôme Tignon, Sukhdeep Dhillon

\section{To cite this version:}

Kenneth Maussang, Anthony Brewer, José Palomo, Jean-Michel Manceau, Raffaele Colombelli, et al.. Echo-Less Photoconductive Antenna Sources for High-Resolution Terahertz Time-Domain Spectroscopy . IEEE Transactions on Terahertz Science and Technology, 2016, 6 (1), pp.6. 10.1109/TTHZ.2015.2504794 . hal-01278208

\section{HAL Id: hal-01278208 \\ https://hal.science/hal-01278208}

Submitted on 23 Feb 2016

HAL is a multi-disciplinary open access archive for the deposit and dissemination of scientific research documents, whether they are published or not. The documents may come from teaching and research institutions in France or abroad, or from public or private research centers.
L'archive ouverte pluridisciplinaire HAL, est destinée au dépôt et à la diffusion de documents scientifiques de niveau recherche, publiés ou non, émanant des établissements d'enseignement et de recherche français ou étrangers, des laboratoires publics ou privés. 


\title{
Echo-less Photoconductive Antenna sources for High-Resolution Terahertz Time-domain Spectroscopy
}

\author{
Kenneth Maussang, Anthony Brewer, José Palomo, Jean-Michel Manceau, Raffaele Colombelli, \\ Isabelle Sagnes, Juliette Mangeney, Jérôme Tignon and Sukhdeep S. Dhillon
}

\begin{abstract}
Interdigitated photoconductive antennas are powerful and easy-to-use sources of terahertz radiation for timeresolved spectroscopy. However, the emission of unwanted echoes, resulting from reflections of the emitted pulse in the antenna substrate, inherently limits the spectroscopic frequency resolution. A novel interdigitated photoconductive antenna that suppresses unwanted echoes from the substrate, without power losses, is proposed and demonstrated. This is realized through a buried metal geometry where a metal plane is placed at a subwavelength thickness below the surface antenna structure and GaAs active layer. In a reflection geometry this effectively eliminates echoes, permitting high resolution spectroscopy to be performed. As a proof-of-principle, the $1_{01}-2_{12}$ and the $2_{12}-3_{03}$ rotational lines of water vapor have been spectrally resolved with the new buried metal antenna, which are unresolvable with a standard antenna. In addition, as no $\mathrm{THz}$ field is lost to the substrate and reflections, the terahertz peak electric field amplitude is enhanced by a factor of three compared to a standard design in the equivalent reflection geometry.
\end{abstract}

Index Terms-Terahertz antenna, terahertz photoconductive sources, terahertz radiation, ultrafast photoconductors, submillimeter wave devices.

\section{INTRODUCTION}

A DVANCES in Terahertz (THz) spectroscopy have resulted in a wide variety of new applications from materials science [1]-[3], to sensing [4], [5]. This opening of applications in the $\mathrm{THz}$ region (from $\sim 100 \mathrm{GHz}$ to $\sim 10 \mathrm{THz}$ ) is due to rapid developments in new technologies and systems. With the emergence of ultrafast lasers, a popular technique is

This work was support by the French National Research Agency (ANR-12NANO-0014 "PhaseLock"). This project has received funding from the European Union's Horizon 2020 research and innovation programme under grant agreement No 665158. Laboratoire Pierre Aigrain is a 'Unité Mixte de Recherche' associated to CNRS (UMR8551), Ecole Normale Supérieure, Université Pierre et Marie Curie (Paris 6) and Université Denis Diderot (Paris 7).

K. Maussang, A. Brewer, J. Palomo, J. Mangeney, J. Tignon, and S. S. Dhillon are with the Laboratoire Pierre Aigrain, Ecole Normale SupérieurePSL Research University, CNRS, Université Pierre et Marie Curie-Sorbonne Universités, Université Denis Diderot-Sorbonne Paris Cité, 24 rue Lhomond, 75231 Paris Cedex 05, France (e-mail: kenneth.maussang@ens.fr).

J.-M. Manceau, and R. Colombelli are with the Institut d'Electronique Fondamentale, CNRS (UMR8622), Bât. 220-Université Paris Sud, 91405, Orsay, France.

I. Sagnes is with the Laboratoire de Photonique et de Nanostructures, CNRS (UPR20), Route de Nozay, 91460, Marcoussis, France.
THz time-domain spectroscopy (TDS). This approach provides broadband spectroscopic capability and overcomes many of technical difficulties traditionally associated with research in the $\mathrm{THz}$ range, such as the requirement for cryogenics for detectors or sources. While other spectroscopic methods are conducted directly in the frequency domain (by frequency sweeping or interferometric measurements), THzTDS provides direct access to the electric field in the timedomain using femtosecond laser pulses for the generation and detection of single cycle $\mathrm{THz}$ pulses. Although a wide range of methods can be used to generate $\mathrm{THz}$ pulses, one of the most common is based on photoconductive antennas [6], [7] that have the advantages of large spectral bandwidth and signal-to-noise ratio, as well as being suited for high frequency electrical modulation, reducing $1 / \mathrm{f}$ noise.

In a typical arrangement, a metallic antenna structure (with anode and cathode) is realized on top of semiconductor substrate via standard photolithography. The antenna is excited with an interband excitation using an ultrafast laser $(\lambda \sim 810 \mathrm{~nm})$, generating carriers which are accelerated by an applied static electric field between the electrodes [6],[7]. The corresponding time-varying current generates electromagnetic pulses in the THz range (see Fig. 1a), one transmitted through the substrate and the other reflected from the front surface. However, owing to the finite thickness of the substrate $(\mathrm{d}$ $500 \mu \mathrm{m})$ and its refractive index contrast with air, the original transmitted pulse is reflected back and forth in the substrate, resulting in 'echoes' i.e. successive $\mathrm{THz}$ pulses separated by the round-trip time, $t=2 d n / c$, where $n$ is the refractive index and $c$ is the speed of light. To avoid the introduction of strong Fabry-Perot oscillations in the spectra as a result of these echoes, the time scans are artificially cut to the range before the first echo, limiting the spectral resolution (proportional to the duration of the time scan). For example, with a photoconductive antenna made from a $500 \mu \mathrm{m}$ thick GaAs wafer and with $n=3.6$ in the $\mathrm{THz}$ range, the first $\mathrm{THz}$ echo arises after only $12 \mathrm{ps}$, limiting the resolution to $\sim 90 \mathrm{GHz}(3$ $\left.\mathrm{cm}^{-1}\right)$. This can restrict certain applications such as high resolution $\mathrm{THz}$ spectroscopy of many polar molecules, where pure rotational spectra typically have linewidths ranging from $0.1 \mathrm{~cm}^{-1}$ to $10 \mathrm{~cm}^{-1}$ (e.g. rotational constant $\mathrm{B}=2 \mathrm{~cm}^{-1}$ for $\mathrm{CO}$ molecule) [8], [9]. Several hardware methods have been proposed to overcome this echo issue but do not completely 
a)

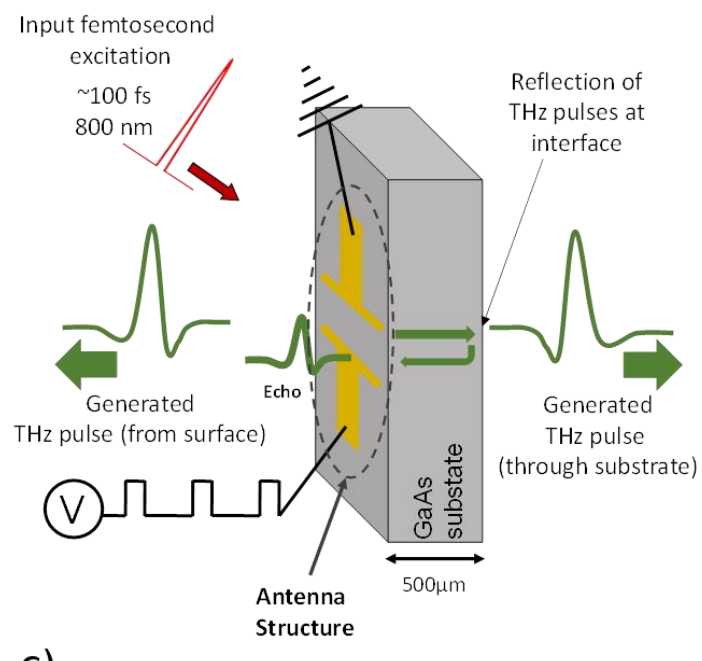

c)

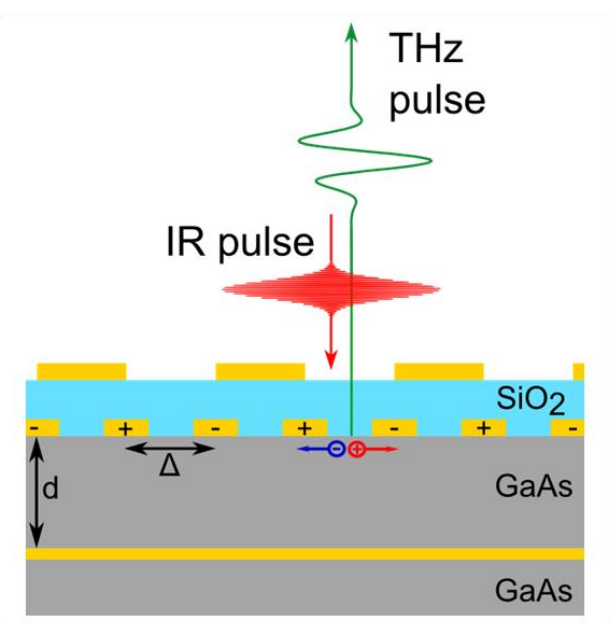

b)

d)
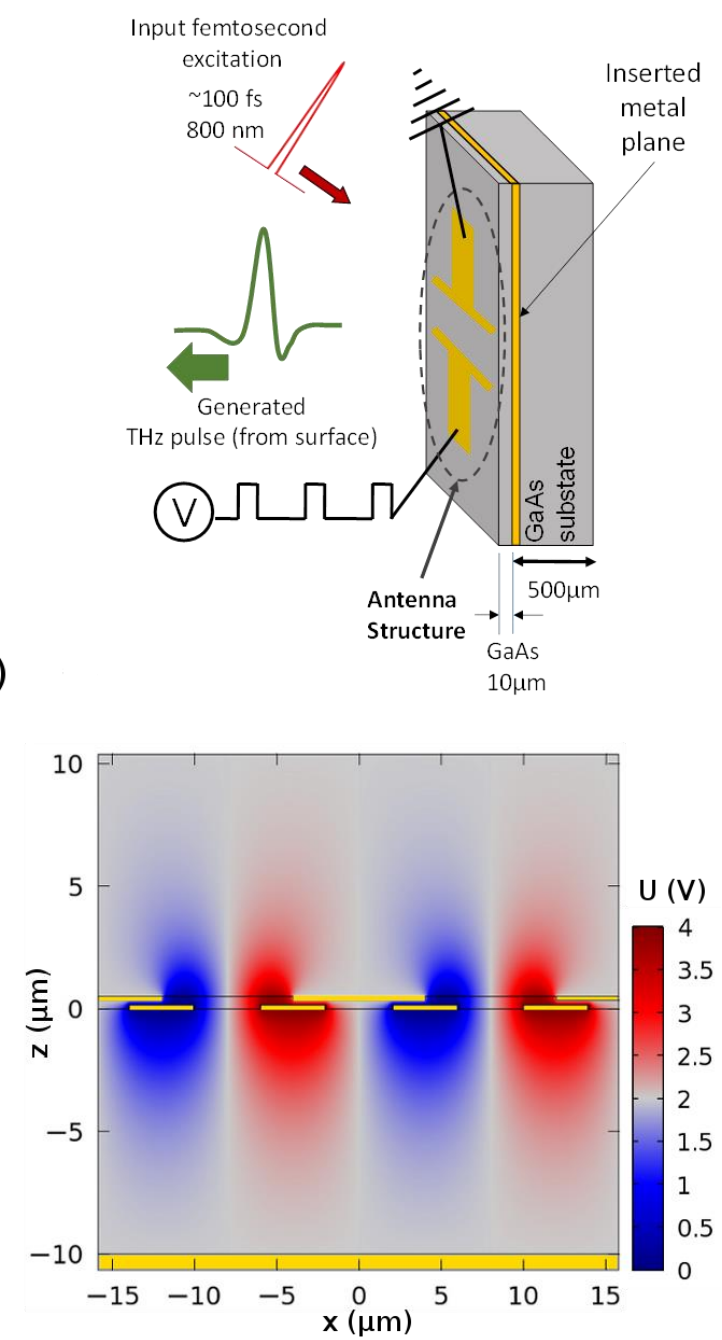

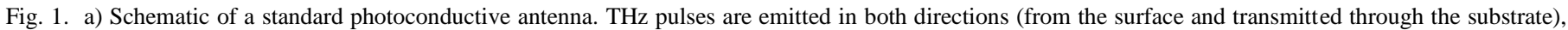

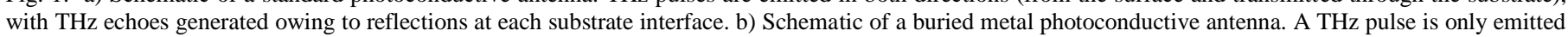

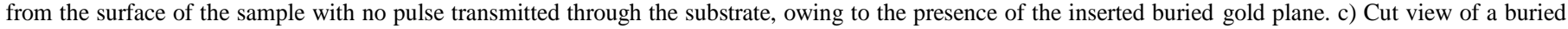

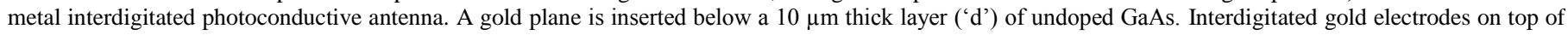

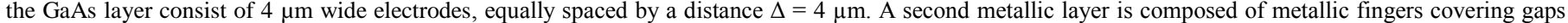

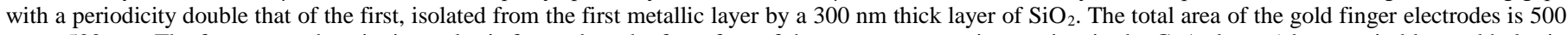

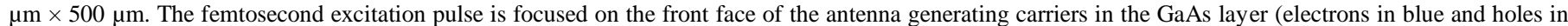
red). The $\mathrm{THz}$ pulse is emitted from the surface. d) Calculated electrical potential $\mathrm{U}$ for an applied voltage of $4 \mathrm{~V}$.

resolve the problem: $\mathrm{THz}$ dielectric anti-reflection coating [10] have been demonstrated but have a narrow bandwidth and thus not adapted for broadband emission; broadband thin metal coatings [11] are difficult to realize and introduce important losses. Alternative approaches consist in numerical methods, either by deconvolution with a reference signal [12], [13], or echo cancelation with a deconvolution algorithm [14], [15]. However, those methods require either a careful calibration of the reference, or an assumption of the dispersion properties of the substrate, respectively. As a consequence, with complex $\mathrm{THz}$ signals [16] these software solutions can add artefacts in the calculated spectrum. A simple method consists simply to realize antennas on thicker substrates but this only displaces the echoes to latter times. Finally, another approach consists in the use of a silicon hemispherical lens of the appropriated thickness manually placed behind the antenna structure that reduces or eliminates the Fabry-Perot effect [17].
However, it has only been demonstrated in the case of small $\mathrm{THz}$ spot size photoconductive antennas (for example ref. [18]) and is not adapted to these large area antennas. This is owing to the large $\mathrm{THz}$ spot size, which does not act as a punctual source and thus does not eliminate the echo.

In this paper, we propose and realize a novel antenna design that intrinsically removes pulse echoes through a unique buried metal geometry, providing a monolithic "on-chip" solution without any mechanical positioning of external elements post processing. Rather than displacing the echo to longer times, we bring the echo to shorter and shorter times by placing a metal plane at a subwavelength distance from the antenna surface. At such distances, the metal plane acts as a cavity and hence effectively eliminates the echo within the corresponding spectral bandwidth. Moreover, as there are no echoes, no power is lost to the substrate leading to a factor of three increase in the $\mathrm{THz}$ field in a reflection geometry. 


\section{BURIED-METAL INTERDIGITATED PHOTOCONDUCTIVE ANTENNA DESIGN}

Fig. $1 \mathrm{~b}$ shows a schematic of the buried metal interdigitated photoconductive (IPC) antenna. Compared to standard antenna device (Fig. 1a), a thin metal plane is inserted between the active GaAs layer (see below for processing details) at a distance $d$ from the surface to reflect the generated THz power with no THz field transmitted through the substrate. Since the transient current photogenerated by the optical excitation pulse has its direction fixed by the interdigitated metallic layers, parallel to the buried gold plane, the structure is similar to an optical Fabry-Perot resonator. The lower longitudinal mode satisfy the resonance condition $d=\lambda / 2$, where $\lambda$ is the wavelength in the active layer. Thus, the distance $d$ has been chosen such that the first cut-off frequency corresponds to the upper limit of the detection bandwidth of the setup (typically $0.1 \mathrm{THz}$ to $4 \mathrm{THz}$ in the setup used). For a generated $\mathrm{THz}$ pulse typically covering this spectral range, corresponding to wavelengths from $830 \mu \mathrm{m}$ to $22 \mu \mathrm{m}$ in GaAs $(n=3.6)$, a layer of $d=10 \mu \mathrm{m}$ is deeply subwavelength and can be considered infinitely thin without any resonant modes. It can be also viewed in the time-domain: the thickness of substrate is chosen to be so small that all echoes are emitted on a time scale smaller than the mean oscillation period of the radiation. The presence of the buried metal favors more intense field emission around the anti-resonant frequency of the cavity, compared to a standard antenna. The bandpass effect of the buried metal antenna and relative spectral response compared to a standard antenna has been investigated through numerical simulations, with COMSOL Multiphysics software (Fig. 2). A floating plane for the buried metal was used as well as a parallel dipole on the top surface of the semiconductor for both antennas. Furthermore, the normalized radiation pattern has been calculated (inset of Fig. 2) which shows an almost identical far field between a standard and buried metal antenna.

The buried metal structure was realized through an epilayer transfer process. The sample was grown by metal organic chemical vapor phase deposition (MOCVD) on a semi-insulating (SI) GaAs wafer. The growth included a GaAs buffer layer, an $\mathrm{AlGaAs}$ etch stop layer (50\% alumimium), followed by the $10 \mu \mathrm{m}$ thick undoped GaAs active layer. After a gold evaporation on top of the wafer, the grown sample was wafer bonded to a gold coated host SI GaAs substrate. The original substrate was then removed with a selective etch based on citric acid. The AlGaAs was etched using a hydrofluoric etch, leaving the GaAs active region exposed with a metal plane $10 \mu \mathrm{m}$ below the surface. The antenna structure was then processed on top of the GaAs surface. An interdigitated geometry [19], [20] was chosen as it provides a large surface area but with a small electrode spacing. This allows efficient collection of the $\mathrm{THz}$ emission without the use of hemi-spherical lenses and low biases to be applied to the structure, respectively (see Fig. 1c). The processing of these interdigitated photoconductive antennas is described in reference [19]. A finger separation of $4 \mu \mathrm{m}$ was used, with 4 $\mu \mathrm{m}$ wide electrodes. Fig. $1 \mathrm{~d}$ shows the simulation of the static field, with virtually no field at the buried metal plane. A 300 nm-thick layer of $\mathrm{SiO}_{2}$ is deposited, allowing the electrical insulation of the gold fingers from the second metal layer. This is composed of metallic fingers covering gaps with a periodicity double that of the first (see Fig. 1c). This permits optical excitation of every second period of the gaps of the first metallization and hence excitation of only one bias field direction. This prevents destructive interferences of the generated THz far field. Fig. 1c shows a schematic cut profile of the realized buried metal antenna. For comparison, an identical interdigitated antenna was also realized on a $500 \mu \mathrm{m}$ thick SI GaAs substrate (i.e. without the buried metal plane).

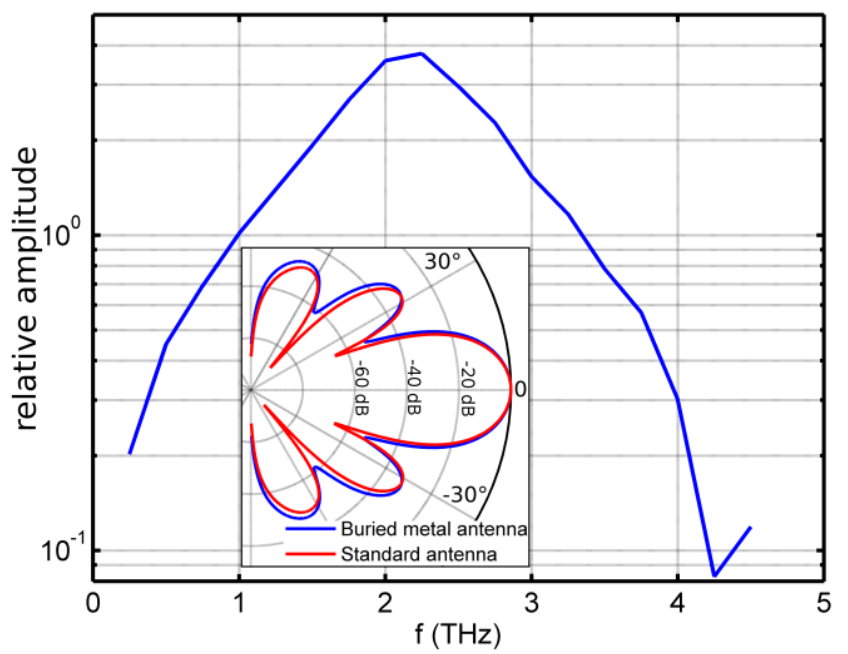

Fig. 2. Numerical simulations of the relative frequency response of a buried metal antenna compared to standard antenna geometry, performed with COMSOL Multiphysics. The buried metal cavity behaves as a bandpass filter with a higher spectral density within 1-3.3 THz window. Inset: simulated radiation diagram of both antennas at $2 \mathrm{THz}$ (normalized response on log scale).

\section{COMPARATIVE PERFORMANCES}

The experimental setup used to measure the emitted $\mathrm{THz}$ field is shown in Fig. 3 and based on a standard THz time domain spectroscopy set-up. Antennas were biased at $4 \mathrm{~V}$ corresponding to an applied electric field of $10 \mathrm{kV} / \mathrm{cm}$. An ultrafast (100 fs pulses) Ti:sapphire oscillator centered at 810 $\mathrm{nm}$ was used to photo-excite carriers in the GaAs active layer. Average powers of $\sim 130 \mathrm{~mW}$ were used. The generated $\mathrm{THz}$ pulses were collected from the front surface of the GaAs active layer for both the buried metal and standard antenna structures in a reflection geometry. Standard electro-optic sampling [21] was used to detect the electric field of the $\mathrm{THz}$ pulses, using a $200 \mu \mathrm{m}$ thick $\langle 110\rangle \mathrm{ZnTe}$ crystal placed on 2 $\mathrm{mm}$ thick host substrate. A mechanical delay line is used to sample the THz ultrafast pulse as a function of time. The THzTDS setup is placed in a dry-air purge chamber (typically < $2 \%$ humidity) to reduce water absorption of $\mathrm{THz}$ radiation.

Fig. 4 shows the measured electric field as a function of time for the buried metal antenna and the standard antenna. While the standard antenna shows the expected echoes separated by $12 \mathrm{ps}$, the buried metal antenna shows that these 


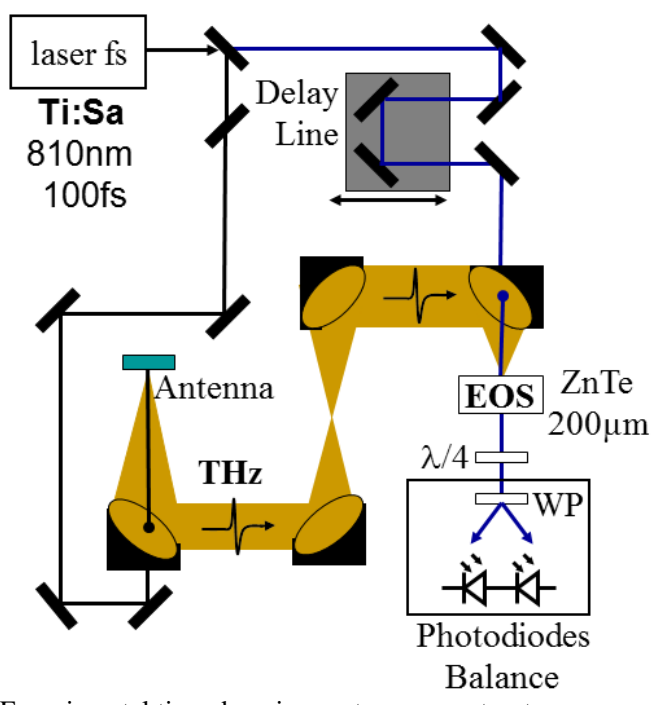

Fig. 3. Experimental time domain spectroscopy setup to measure the $\mathrm{THz}$ field emitted by the antennas. An Ti:Sapphire oscillator (100fs, $810 \mathrm{~nm}$ ) excites the biased antenna. The $\mathrm{THz}$ field is collected with parabolic mirrors and focused onto a ZnTe crystal for electro-optic sampling (EOS). The induced polarization change is measured with a photodiode balance,

echoes are totally eliminated. An extra cycle in the first pulse is observed for the buried metal antenna, corresponding to a slightly reduced bandwidth (see below) attributed to the etalon effect between the buried and surface metal. At $45 \mathrm{ps}$, a reflection is present in both antenna devices owing to the 2.2 $\mathrm{mm}$ thickness of the ZnTe crystal used for detection. The inset shows an enhanced view of the time traces, showing no echoes for the buried metal antenna, with the observed oscillations due to residual absorption by water. No discontinuity is observed in the time trace around the principal pulse, owing to the sub-wavelength cavity, showing that the echo is eliminated in the experimental frequency range. As well as removing the echoes, the entire field is concentrated in the first and unique pulse as the $\mathrm{THz}$ pulse does not undergo any reflections in the antenna substrate. This provides more intense pulses, with peak and peak-to-peak amplitudes three and four times, respectively, greater than produced with the standard antennas. (The radiation efficiency of these photoconductive antennas normally defined as the $\mathrm{THz}$ output power divided by the optical input power is in the typical range $10^{-5}-10^{-4}$ ). The peak spectral density of buried metal is increased by a factor 2 compared to a standard antenna (see Fig. 5). (The response of the standard antenna was also measured in a normal transmission geometry, where the peak spectral density is $60 \%$ higher compared to reflection geometry owing to the higher refractive index of the substrate. Therefore a buried metal antenna still provides a higher peak spectral density).

The $\mathrm{THz}$ pulse can be easily depicted in the frequencydomain by taking the fast Fourier transform (FFT) of the timedomain trace. Fig. 5a) shows the spectrum for the standard and buried metal antenna. The FFT operation was performed with a rectangular window ending just before the echo from the $\mathrm{ZnTe}$ crystal $(\mathrm{t}=44 \mathrm{ps})$ for both antennas (blue and red curves), and just before the first echo at $t=8$ ps for the standard antenna (black), and a standard zero-padding procedure. As expected, the spectrum of the standard antenna with the FFT of the entire time trace shows strong Fabry-Perot oscillations as a result of echoes from the substrate. The spectrum of the standard antenna with the smaller FFT window avoids the spectral oscillations but shows a considerably reduced spectral resolution. However, the buried metal antenna spectrum has a high resolution with many water absorption peaks resolved without the presence of the spectral oscillations.

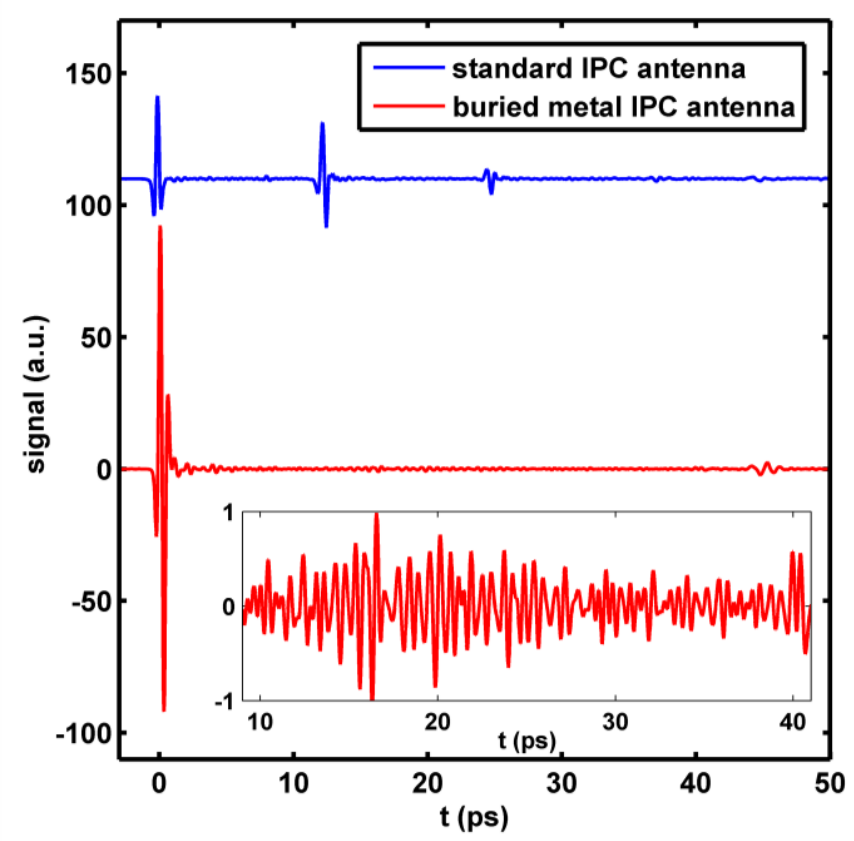

Fig. 4. Time domain trace of $\mathrm{THz}$ electric field obtained with a standard antenna (blue line) and a buried metal antenna (red line), with the same applied bias field, both interdigitated photoconductive (IPC) antennas. The peak amplitude of the buried antenna is 3 times higher than a standard interdigitated antenna. Inset: enhanced view of the y-axis showing oscillations on the antenna traces resulting from residual water in TDS setup chamber.

To highlight the increased spectral resolution, Fig. 5b) shows a zoom of the 1.5 to $2 \mathrm{THz}$ spectral window that shows certain absorption features owing to the residual water vapor in the purged chamber. The normalized spectrum modulus and spectral phase of each antenna has been plotted and clearly shows that the spectral features are narrower in the case of the buried metal antenna. While with the usual antenna design only a single broad peak is visible, the buried metal antenna can resolve unambiguously two intense lines at $1.670 \mathrm{THz}$ and $1.719 \mathrm{THz}$ corresponding to the $1_{01}-2_{12}$ and the $2_{12}-3_{03}$ rotational lines, which agree well with database measurements at $1.669904 \mathrm{THz}$ and $1.716769 \mathrm{THz}$ [22], [23], respectively. Weaker lines are also visible at $1.868 \mathrm{THz}$ and $1.923 \mathrm{THz}$, corresponding to the $5_{23}-5_{32}$ and the $3_{13}-3_{22}$ rotational lines (measured at $1.867748 \mathrm{THz}$ and $1.919359 \mathrm{THz}$ in databases). Therefore, a $47 \mathrm{GHz}$ line splitting can be easily measured with such an 'echo-free' THz source. The smallest spectral splitting that could be resolved was $20 \mathrm{GHz}$. Moreover, the spectral phase $\phi(\omega)$ also exhibits clear dispersive profiles at the corresponding absorption lines. This unique property of timedomain spectroscopy to provide information both in amplitude and in phase permits to access to the complex index of refraction of the studied material without requiring the application of Kramers-Kronig type relationship [24]. 
a)

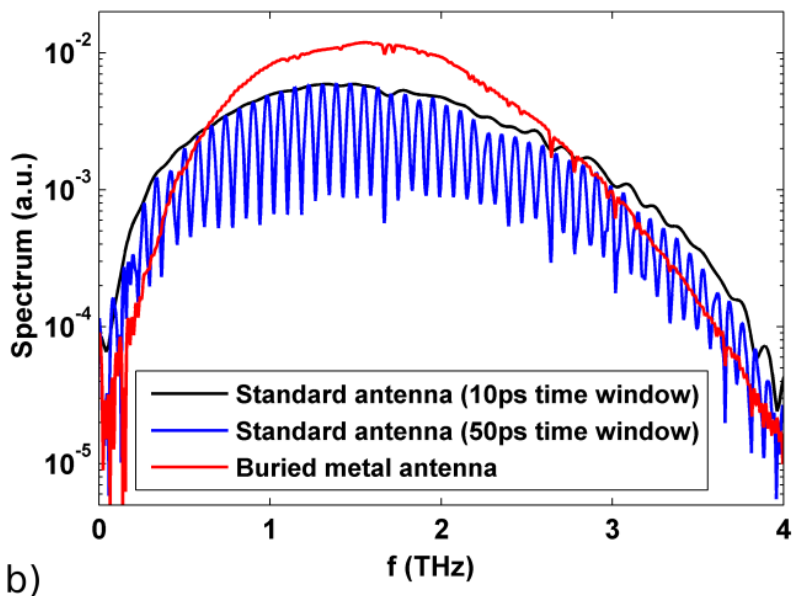

b)
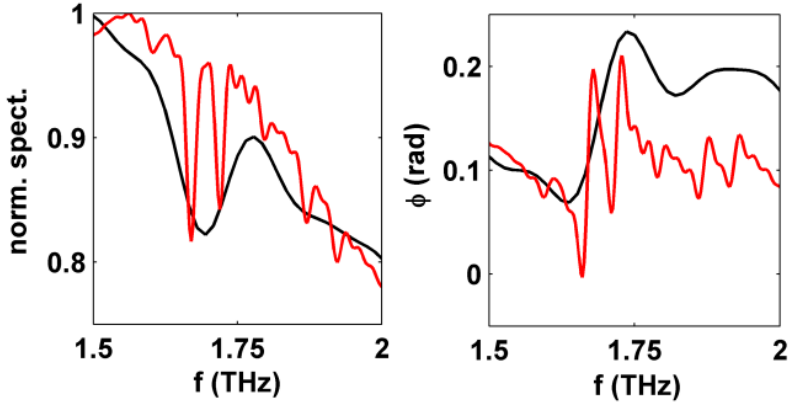

Fig. 5. a) THz spectral field from the FFT of the time domain traces (log profile). The peak spectral density of buried metal antenna is 2 times higher than the standard interdigitated photoconductive antenna. Relative amplitude at low frequencies $(<0.5 \mathrm{THz})$ and high frequencies $(>2.8 \mathrm{THz})$ are lower for the buried metal antenna owing to etalon effects of the buried metal and antenna surface. b) Enhanced view of the normalized spectral field (left figure) in the 1.5-2 THz range; $1_{01}-2_{12}, 2_{12}-3_{03}, 5_{23}-5_{32}$ and $3_{13}$ - $3_{22}$ rotational lines of water are resolved only in the case of the buried metal antenna. The spectral phase $\phi(\omega)$ (right figure) is also plotted, showing a dispersive profile at the absorption resonances.

\section{CONCLUSION}

In conclusion, we have demonstrated the possibility to intrinsically suppress unwanted echoes arising from reflections of $\mathrm{THz}$ pulses in the substrate of $\mathrm{THz}$ antennas. The realized monolithic device consists in a buried metal approach that is based on standard epilayer transfer and fabrication techniques. The resolution of a standard THz-TDS setup has been demonstrated to reach the tens of $\mathrm{GHz}$ range, only limited by the echo in the electro-optic crystal. With a similar concept applied to low temperature GaAs to realize an antenna for detection, even greater resolution would be achieved that would be limited only by the repetition rate of the femtosecond laser $(80 \mathrm{MHz})$. In addition, since the energy previously distributed in echoes is fully concentrated in the main and unique pulse, the peak amplitude of the emitted pulse is three times higher compared to standard interdigitated photoconductive antenna design. Further work will also include optimization of the GaAs active layer thickness to increase the spectral bandwidth.

\section{REFERENCES}

[1] M. Tonouchi, "Cutting-edge terahertz technology," Nature Photonics, vol. 1, pp. 97-105, Feb. 2007.

[2] Y. C. Shen, "Terahertz Time-Domain Spectroscopy and Imaging," J. Electr. Electron. Syst., vol. 3, no. 1, Dec. 2013.

[3] M. Beard, G. Turner, and C. Schmuttenmaer, "Terahertz spectroscopy," J. Phys. Chem. B, vol. 106, pp. 7146-7159, Jun. 2002.

[4] D. M. Mittleman, R. H. Jacobsen, R. Neelamani, R. G. Baraniuk, and M. C. Nuss, "Gas sensing using terahertz time-domain spectroscopy," Appl. Phys. B, vol. 67, pp. 379-390, Feb. 1998.

[5] D. Mittleman, Sensing with $\mathrm{THz}$ Radiation. Ed. Heidelberg: Springer, 2002

[6] P. Uhd Jepsen, R. H. Jacobsen, and S. R. Keiding, "Generation and detection of terahertz pulses from biased semiconductor antennas," J. Opt. Soc. Am. B, vol. 13, pp. 2424-2436, Nov. 1996.

[7] Y. C. Shen, P. C. Updadhya, E. H. Linfield, H. E. Beere, A. G. Davies, "Ultrabroadband terahertz radiation from low-temperaturegrown GaAs photoconductive emitters," Appl. Phys. Lett., vol. 83, pp. 3117-3119, Oct. 2003.

[8] G. Herzberg, Molecular Spectra \& Molecular Structure. Vol. III: Polyatomic Molecules. Ed. New-York: D. Van Nostrand Reinhold Co. cop., 1966.

[9] G. Herzberg, Molecular Spectra \& Molecular Structure. Vol. IV: Constants of diatomic. Ed. New-York: D. Van Nostrand Reinhold Co. cop., 1966.

[10] A. J. Gatesman, J. Waldman, M. Ji, C. Musante, and S. Yngvesson, "An anti-reflection coating for silicon optics at terahertz frequencies," IEEE Microw. Guided Wave Lett., vol. 10, pp. 264266, Jul. 2000

[11] A. Thoman, A. Kern, H. Helm, and Markus Walther, "Nanostructured gold films broadband terahertz antireflection coatings," Phys. Rev. B, vol. 77, no. 195405, May 2008.

[12] J. R. Fletcher, G. P. Swift, D. C. Dai, J. M. Chamberlain, and P. C. Upadhaya, "Pulsed Terahertz Signal Reconstruction," J. Appl. Phys., vol. 102, no. 113105, Dec. 2007.

[13] O. Hirsch, P. Alexander, and L. Gladden, "Techniques for cancellation of interfering multiple reflections in terahertz timedomain measurements," Microelectron. J., vol. 39, pp. 841-848, May 2008.

[14] A. Heidari, M. Neshat, D. Saeedkia, and S. Safavi-Naeini, "Signal recovery in pulsed terahertz integrated circuits," Progress In Electromagnetics Research, vol. 107, pp. 269-292, Aug. 2010.

[15] A. Redo-Sanchez, and X.-C. Zhang, "Self-referenced method for terahertz wave time-domain spectroscopy," Opt. Lett., vol. 36, pp. 3308-3310, Sep. 2011.

[16] D. Oustinov, N. Jukam, R. Rungsawang, J. Madéo, S. Barbieri, P. Filloux, C. Sirtori, X. Marcadet, J. Tignon, and S. Dhillon, "Phase seeding of a terahertz quantum cascade laser," Nature Comm., vol. 1, no. 69, Sep. 2010.

[17] M. C. Nuss and J. Orenstein, "Terahertz time-domain spectroscopy," Millimeter and Submillimeter Wave Spectroscopy of Solids, Topics in Applied Physics, vol. 74, pp. 7-50, 1998.

[18] S.-G. Park, M. R. Melloch and A. M. Weiner, "Comparison of terahertz waveforms measured by electro-optic and photoconductive sampling," Appl. Phys. Lett., vol. 73, pp. 3184-3186, 1998.

[19] A. Dreyhaupt, S. Winnerl, T. Dekorsy, and M. Helm, "Highintensity terahertz radiation from a microstructured large area photoconductor," Appl. Phys. Lett., vol. 86, no. 121114, Mar. 2005.

[20] J. Madéo, N. Jukam, D. Oustinov, M. Rosticher, R. Rungsawang, J. Tignon, and S. S. Dhillon, "Frequency tunable terahertz interdigitated photoconductive antennas," Elec. Lett., vol. 46, pp. 611-613, Apr. 2010.

[21] Q. Wu, and X.-C. Zhang, "Free-space electro-optic sampling of terahertz beams," Appl. Phys. Lett., vol. 67, pp. 3523-3525. 1995.

[22] X. Xin, H. Altan, A. Saint, D. Matten, and R. R. Alfano, "Terahertz absorption spectrum of para and ortho water vapors at different humidities at room temperature," J. Appl. Phys., vol. 100, no. 094905, Nov. 2006.

[23] values extracted from Nasa internet database http://spec.jpl.nasa.gov

[24] V. Lucarini, J.J. Saarinen, K.-E. Peiponen, and E.M. Vartiainen, Kramers-Kronig Relations in Optical Materials Research. Ed. Heidelberg: Springer, 2005. 


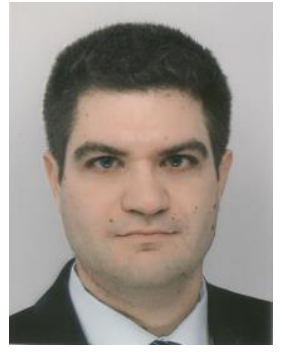

Kenneth Maussang received his M.S. degree in 2006, and his Ph.D. degree in 2010 from Université Pierre et Marie Curie, Paris, France, in quantum physics. $\mathrm{He}$ performed his $\mathrm{PhD}$ in Laboratoire Kastler Brossel (ENS, Université Pierre et Marie Curie, CNRS), where his research dealt with atomic physics, more specifically degenerated ultracold quantum gases manipulated with atomchips technologies, and included studies of atomic squeezed states in Bose-Einstein condensates. Since 2011, he is a postdoctoral fellow in Laboratoire Pierre Aigrain (ENS, Université Pierre et Marie Curie, Université Denis Diderot, CNRS), where his research is oriented to terahertz technologies, including time-domain spectroscopy and quantum cascade lasers, applied to condensed matter physics.

Anthony Brewer is the founder of agAlytics, a technology startup in Brisbane, Australia. His current research is in labon-a-chip technologies for the infield analysis of agricultural samples, in particular quantifying nutrients in soil and plant tissue. His previous work was in the Terahertz sciences based on a postdoctoral research position at the Ecole Normale Supérieure in Paris, France and a PhD from the University of Cambridge, UK.

José Palomo received a B.S. in applied physics in 1996 from Université de Versailles-Saint-Quentin-en-Yvelines. He first worked in the clean room facility of the Institut d'Electronique Fondamentale of Universite Paris-Sud. $\mathrm{He}$ is currently working as technical co-responsible of the clean room facility of the Ecole Normale Superieure, where he works in collaboration with different research team in the realization of micro and nano devices.

Jean-Michel Manceau Jean-Michel Manceau is currently at the Insitut d'Electronique Fondamentale in Orsay, France. He is a researcher of the "Centre National de la Recherche Scientifique" (CNRS). His current research activities are centered around intersubband polaritonic devices in the mid and far infrared. His previous work has been based on a postdoctoral period at University Paris VII as well as $\mathrm{PhD}$ from the University of Crete in Greece where he worked on terahertz time domain spectroscopy.

Raffaele Colombelli received the Ph.D. degree in physics from the Scuola Normale Superiore, Pisa, Italy, in 1999. He then joined Bell Laboratories, Murray Hill, NJ, where he focused on long-wavelength quantum cascade lasers, and the application of photonic technology to unipolar devices. In 2003, he joined the "Centre National de la Recherche Scientifique" (CNRS) at Institut d'Electronique Fondamentale (Orsay, FRANCE), where he is research director. His current research interests include the development of terahertz semiconductor lasers, the application of antenna and circuital techniques to $\mathrm{THz}$ devices, and intersubband polaritons.

Isabelle Sagnes is currently at the Laboratoire de Photonique et Nanostructures (LPN) in Marcoussis, France. She is a senior researcher of the "Centre National de la Recherche Scientifique" (CNRS). She received the Ph.D. degree in physics from the University Joseph Fourier, Grenoble, France, in 1994, for her work in electro-optical properties of epitaxial heterostructures on silicon. She joined the CNET Laboratory, Bagneux, France, in 1997, as a Research Engineer in semiconductor III-V compounds. In 1999, she joined the CNRS at LPN which move from Bagneux to Marcoussis in 2001. Her research interests include MOCVD growth of complex heterostructures on $\mathrm{GaAs}$ and InP substrates, MOCVD growth of quantum dots on GaAs and InP substrates, heteroepitaxy of $\mathrm{GaAs}$ on $\mathrm{Ge} / \mathrm{Si}$ virtual substrate, and III-V device technology as the processing of photonic crystals.

Juliette Mangeney did her PhD at University Paris-Sud 11 and at CNET where she studied ultrafast saturable absorbers for telecommunication applications. After 1-year post-doc position at University Paris-Sud 11, she joined in 2001 CNRS as member of staff of Laboratory IEF at University Paris-Sud 11. Her scientific work was devoted to the development of femtosecond and $\mathrm{THz}$ optoelectronic metrology and to the study of non-equilibrium carrier dynamics in ultrafast semiconductors. In 2009, she headed the research group on $\mathrm{THz}$ optoelectronic at Laboratory IEF. In 2012, she joined the group Ultrafast $\mathrm{THz}$ Spectrocopy in Laboratoire Pierre Aigrain at Ecole Normale Supérieure (France) where she heads activities on carbon-based materials for $\mathrm{THz}$ technology and on ultrabroadband $\mathrm{THz}$ spectroscopy.

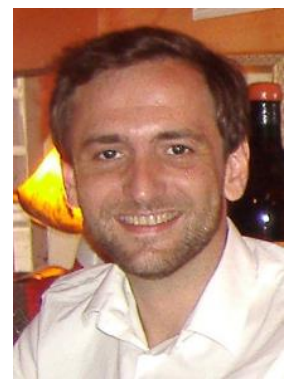

Jerome Tignon is Professor at University Pierre et Marie Curie and Ecole Normale Supérieure in Paris. He received his $\mathrm{PhD}$ at ENS in 1996. He then joined the Lawrence Berkeley National Laboratory in Berkeley, for a 2 years' post-doc position in D.S. Chemla's group, conducting research on ultra-fast spectroscopy of III-V semiconductors. In 1998 he joined the Laboratoire Pierre Aigrain at ENS where he first developed the activity on coherent and nonlinear optics for investigating the dynamics of electronic excitations in semiconductor nanostructures. His main contributions in this context concern the study of the coherent dynamics of polaritons and the optical parametric oscillation in semiconductor microcavities. In 2006, Jerome Tignon created the "ultra-fast $\mathrm{THz}$ spectroscopy group" in Laboratoire Pierre Aigrain at ENS, which he continues to head at the present time. He developed the study of the dynamics of THz-quantum cascade lasers, spin excitations in diluted magnetic semiconductors, and carbon-based structures.

Sukhdeep S. Dhillon is currently at the Ecole Normale Supérieure in Paris, France. He is a senior researcher of the "Centre National de la Recherche Scientifique" (CNRS). His current research activities are centred around quantum cascade lasers and in particular their ultrafast and nonlinear properties. His previous work has been based on a postdoctoral period at Thales Research and Technology and University Paris VII as well as $\mathrm{PhD}$ from the University of Cambridge, UK. 